\title{
Use of Individually Scaled Versus Normatively Scaled Predictor Cues in Policy-Capturing Research
}

\author{
Bill Cotton \\ University of California, Riverside \\ Rick Jacobs \\ The Pennsylvania State University \\ Janet Grogan \\ University of California, Riverside
}

Current policy-capturing models scale the levels of the predictor cues on the basis of normative data collected on a group of subjects. Two studies were conducted to determine whether the performance of these models would be improved by scaling cue values individually for each decision maker. The results of these studies confirmed the hypothesis that the scaling of stimulus cues for each decision maker results in a judgment model that is much more successful in reproducing the decision maker's responses than models employing the same cue scales for all decision makers. Additionally, it was found that the relative performances of models based on regression weights and those models that employ weights generated by the subject are heavily dependent on methodological variables. It is concluded that if there is to be an understanding of the way people utilize information, not only must there be concern about variable weighting, but there must also be consideration of the subjective experience the individual decision maker has with respect to the levels of each variable.

Whether selecting an automobile or evaluating the relative merits of applicants to graduate school, people make decisions mediated by processes that cannot be directly observed. A series of research investigations have attempted to generate predictive models of these decision processes across individuals at the group level (Slovic, Fischhoff, \& Lichtenstein, 1977). A second group of studies has tried to capture the policy of individual decision

APPLIED PSYCHOLOGICAL MEASUREMENT

Vol. 7, No. 2, Spring 1983, pp. 159-171

(C) Copyright 1983 Applied Psychological Measurement Inc. 0146-6216/83/020159-13\$1.65 makers (Wiggins, 1973). Almost all of these studies have been conducted in controlled environments in which the decision maker is provided with several pieces of information, or cues, which must be integrated and a resultant decision produced. Although this paper will evaluate the methodology employed in most policy-capturing studies, the findings are relevant to the more general situation in which behavior is to be explained in terms of a variety of situational cues.

The end goal of any policy-capturing investigation is to achieve a descriptive model of the way in which information is used to produce a judgment about a set of environmental stimuli. Much of this research has tested the adequacy of linear models of decision processes that assume a judgment, or criterion, results from an additive sum of the series of cues multiplied by their respective weights. Thus, according to this model, a given judgment $Y_{\mathrm{J}}^{\prime}$ can be represented as shown in Equation 1.

$\mathrm{Y}_{\mathrm{j}}^{\prime}=\mathrm{B}_{1} \mathrm{X}_{1}+\mathrm{B}_{2} \mathrm{X}_{2}+\ldots+\mathrm{B}_{\mathrm{i}} \mathrm{X}_{\mathrm{i}}$

In this model the $X_{1}$ 's represent the magnitude of the predictor cues, and the $B_{1}$ 's the weight associated with each cue.

Two procedures are typically employed to obtain estimates of the $B_{1}$ 's. Perhaps the most obvious way to determine the judgment strategy is simply to ask the decision maker to numerically estimate the relative importance of the stimulus cues. Hoffman (1960) required subjects to distribute 100 points 
among the various stimulus cues in a manner that reflected the relative importance of the cue. Next, the number of points assigned to each cue became the $B_{1}$ parameters in Equation 1. These values are often referred to as subjective weights. A second estimate of the weight of the predictor cues can be obtained by a least-squares regression analysis of the decision outcomes as a function of the predictor cues. The procedure produces statistical estimates of the $B_{1}$ parameters and these values can also be used as weights in Equation 1.

The adequacy of the model based on the subjective weighting strategy can be contrasted with that of the model based on the weighting strategy determined by regression analysis. This comparison can be evaluated in terms of the squared zeroorder correlations between the actual judgment produced and those predicted by the regression and subjective weight models.

Several studies have compared the percentage of decision variance accounted for by the subjective and regression weight models when both models are constructed and tested on a single sample of judgments (i.e., Hoffman, 1960; Slovic, 1969; Slovic, Fleissner, \& Bauman, 1972; Summers, Taliaferro, \& Fletcher, 1970). Based on the results of these studies that have shown different weighting profiles between subjectively and statistically based estimates and greater predictability for the statistical model, many researchers have accepted the notion that people perform poorly in describing their judgment strategies (Permut, 1973; Slovic, 1969; Slovic et al., 1972; Slovic \& Lichtenstein, 1971; Summers et al., 1970; Zedeck, 1977). For example, Summers et al. (1970) concluded that "'Ss' subjective weights failed to reflect the differential cue weighting indicated by an objective analysis of Ss' responses ... [and] neither S's quantitative estimates of his cue weighting nor his verbal description is likely to convey an accurate account of his policy" (p. 250).

However, the interpretation of these results is questionable when it is considered that the leastsquares regression analysis maximizes the fit between the predictors and criterion, including a capitalization on chance. Thus, it is to be expected that the magnitude of the multiple correlation coef- ficient will be spuriously inflated as a function of the number of parameters statistically estimated (which, of course, favors the regression model that estimates a parameter for each predictor cue in contrast to the subjective weight model, for which no parameters are statistically estimated). Thus, a proper comparison between the two models must use multiple correlation coefficients corrected for shrinkage (Darlington, 1968) or derived from crossvalidated data (Kerlinger \& Pedhazur, 1973). The results of several recent studies employing one or the other of these techniques to obtain an unbiased estimate of degree of linear fit have reported much smaller differences between the subjective and regression weight models in terms of decision variance explained (Cook \& Stewart, 1975; Jacobs \& Cotton, 1979; Nysted \& Magnusson, 1975; Schmitt, 1978).

If the emphasis of research into the discrepancies between subjective and statistical models of decision making are considered along two dimensions, predictability (or product) and understanding (or process), as has been suggested by Anderson and Shanteau (1977), then the above description of research results can be summarized rather succinctly. Studies involving non-cross-validated statistical models and subjective models show a difference in predictability or product (contrasting $R^{2}$ ), as well as in the explication of the decision strategy employed by the subject or process (comparing the profile of weights). When the statistical model is corrected for sample specificity, the superiority in product is reduced. However, even when cross validated, the scheme of variable weighting or process by which the decision is arrived at still varies within persons from the statistical model to the subjective model. That is, for the statistical model it is generally true that a small subset of the variables accounts for most of the predictable decision variance (Jacobs \& Cotton, 1979; Slovic \& Lichtenstein, 1971; Zedeck, 1977). This is in sharp contrast to the weighting schema decision makers claim to be using when directly questioned about decision processes. Decision makers' self-reports reflect a comparatively larger subset of the predictor cues entering into the decision process (Slovic \& Lichtenstein, 1971; Zedeck, 1977). 
Of particular interest is why these separate descriptions of decision-making process differ. This would seem to be one of the crucial issues to be addressed by policy-capturing researchers. Schmitt and Levine (1977) have suggested that rather than continuing to develop models that better reproduce the decision maker's judgments, an emphasis should be placed on understanding the differences between the two descriptions of decision policy, since "research directed to the perceived importance of information and the conformance between that perceived importance and statistical weights may be far more rewarding in terms of understanding the judgmental process"' (p. 28). Clearly, the validity of any conclusions drawn about either the product or process dimensions must depend on the adequacy of the methodology employed to model the judgment strategy.

Conceptually, policy-capturing research has been narrowly defined. Its primary focus has been on determining the weighting strategies that an individual attaches to given cues without consideration for a more basic issue. Specifically, the research has failed to take into account the preceding subjective experience associated with that cue. Methodologically, an emphasis has been placed on a quantitative specification of the stimulus cues in terms of a physical rather than a psychological continuum. For instance, an experiment may attempt to model how an individual weights cues of yearly salary $X_{1}$ and work group size $X_{2}$ in terms of judgments of attractiveness of a hypothetical employment position. The unstated assumption of such a study is that rated attractiveness of the job is linearly related to larger salaries (scaled in number of dollars) and larger (or smaller) work groups (scaled in terms of number of people). Consider an individual who requires a minimum of $\$ 14,500$ to maintain his/her current lifestyle and prefers a medium-sized work group. In a study where yearly salary ranges from $\$ 12,000$ to $\$ 18,000$, and group size ranges from working with two people to working with ten others, the use of the a priori quantitative rather than the individual's interpretation of the values of the predictor cues will more than likely result in an inappropriate model of an individual's decision process owing to the nonlinear relationship between the scale values of salary and work group size and the individual's response to these factors.

Certainly, regression analysis can accommodate such nonlinear transformations between explicit and subjective stimulus values. For instance, additional terms can be included in the model and it will be reasonably predictive of the decision maker's judgments. However, such a model is inadequate in two respects. First, the linear trend associated with salary only approximates the decision maker's subjective experience of the salary cue. Second, since the ratio between the number of observations and predictors is usually less than optimal in policycapturing research, the addition of relevant higher order terms (e.g., $\mathrm{B}_{3} \mathrm{X}_{2}^{2}$ into the linear model for a given sample size will result in an increase in the instability of the beta weights (Nunnally, 1967). Even if such methodological problems can be overcome, the question still remains whether a model that includes such higher order terms really describes the decision processes.

Alternatively, rather than representing the transformation between the a priori and subjective stimulus values by additional terms in the decision model, a determination of the subjective stimulus values can be generated prior to empirical analysis. For instance Zedeck (1977) used a simple ordinal procedure to scale the five levels for six predictor variables. These scaled stimulus values were then employed as predictors in a policy-capturing study. Although such a procedure is preferable to one that employs arbitrary stimulus values, it is deficient to the extent that the stimulus values experienced by an individual decision maker deviate from the group or normatively scaled values provided by the experimenter. Since policy-capturing research attempts description at the level of the individual, it is imperative that the individual decision maker's subjective experience of the stimulus cues is taken into account.

To summarize, if the goal is to develop a decision model that simulates the judgments of a decision maker, then the cue scale upon which the model is based must be determined individually for each decision maker. Anderson (1972) has emphasized the same point in stating that "an under- 
standing of the decision process clearly must be in terms of the subjective values of the judge"' ( $p$. 98). Without the specification of the subjective nature of the stimulus cues, the relative importance, or weights, of the stimulus cues cannot be accurately determined. The problem, of course, is to determine these subjective scale values. The studies presented in this paper will compare two different methods of estimating these subjective stimulus scale values and will contrast the efficiency of decision models employing these values with that of models employing traditional normative scaling procedures.

\section{Experiment 1}

An experiment was designed to determine whether a first-order approximation of individual subjective stimulus values would result in a more predictive model of the decision strategy than models employing group or normative scaling. The manner by which these subjective stimulus estimates might be obtained was suggested by Anderson's theory of functional measurement $(1970,1971,1974)$. The logic for the scaling procedure is as follows. It is assumed that the individual is producing judgments according to the additive model specified in Equation 1 but that no a priori information is available about the subjective experience associated with the various levels of a given predictor cue. However, all other things being equal, the more positive the experience associated with a level of a predictor cue, the more favorable the resultant judgment should be. Thus, one way estimates of subjective scale value can be obtained is by taking each predictor dimension, one by one, and calculating the mean judgment obtained for each level of that cue dimension. These scaled subjective stimulus values for each predictor can be used as the predictor cues in the modeling of the judgmental process. Inherent in this conceptualization of the problem is the possibility that the differences in the performance of the subjective versus statistical weighting scheme may result from a difference between the individual's perceived stimulus values and the values attached to the stimuli by the experimenter. If this is the case, the performance of the statistically de- termined weighting model may differ from the subjective weighting model only as a result of the former model's failure to adequately specify the appropriate relationship between the predictor cues and the criterion.

To test this possibility, two research hypotheses were entertained. First, decision models using individually determined subjective predictor cue scales will more reliably predict the decision maker's judgments than models employing normative cue scaling. Next, the difference in the description of the decision process (as represented by a profile of weights) between the subjective and regression models will be reduced when the regression model employs individually scaled rather than normatively scaled predictor cues. An experiment was conducted to determine whether these scaled stimulus values would produce a more appropriate model of the individual's decision-making process than the often used normative values (e.g., Zedeck, 1977).

\section{Method}

Subjects. Subjects for this study were recruited from psychology courses requiring research participation. In all, 47 students participated in both test and retest portions of the experiment. Each subject was given 2 hours credit for research participation.

Procedure. Subjects were run in groups. Each subject received a booklet containing a set of instructions and stimulus materials. An experimenter was present if the nature of the experimental task required further elaboration. Stimuli consisted of paragraphs developed by Zedeck (1977). Each subject rated 100 paragraphs describing hypothetical employment positions on a 1 to 7 desirability dimension. Each paragraph contained one of five levels for each of the six factors or predictors. The six predictors were (1) opportunity for personal growth and development, (2) opportunity for advancement, (3) size of work group, (4) salary, (5) flexibility in working requirements, and (6) duration of assigned projects. The distribution of values for each stimulus dimension was approximately normal, and the intercorrelations among predictor pairs were approximately zero (Zedeck, 1977). After rating the stimulus paragraphs, each subject was 
required to allocate a total of 100 points to the six predictors according to the relative importance of each predictor in reaching the rating of desirability. These values are later referred to as subjective weights. Seven days after the initial testing phase, the subjects were required to return and once again rate the booklet of 100 paragraphs.

\section{Data Analysis}

Stimulus scaling. Two different methods were used to assign numerical scale values to the descriptive labels corresponding to the five magnitude levels of each predictor dimension. In the first method scale values were derived individually for each subject by taking each subject's 100 criterion ratings and by calculating the average rating for each level of each predictor. Each predictor dimension was' scaled by averaging over all values of the other five predictor variables for each level of the dimension being scaled. The second set of stimulus values used were those of Zedeck's (1977) normative scale. These are called normative scale values because they are average values obtained from a group of subjects by Zedeck (1977); the same group values were used for all subjects in the present experiment.

Variable weighting. The next step in the experiment was to calculate a least squares multiple regression analysis for each subject using the normative cue values as predictors (this model is referred to as the Normative Regression Model) and again with the individually scaled cue dimensions as predictors (Functional Regression Model). These calculations were carried out on the data from the first testing, and the resulting sets of beta weights were used to predict retest responses and to estimate the relative importance of the predictors.

Two other models were generated by employing the subjective weights with the normative cue scales (Normative Subjective Model) and the scaled predictor cues (Functional Subjective Model). The four models were then used to predict the ratings associated with each paragraph in the retest portion of the experiment. For each subject the correlations between four sets of models based on predicted values and the actual retest ratings were calculated and the zero-order correlations noted. Note that using Zedeck's stimulus paragraphs required employing a test-retest rather than a cross-validation procedure. Although cross-validation would have allowed the results of the experiment to be generalized beyond a particular set of stimulus paragraphs, both procedures provide a satisfactory estimate of the actual fit of a model after correction for shrinkage.

\section{Results and Discussion}

Figure 1 shows the mean proportion of decision variance accounted for by each of the four models. For the tests of significance reported below, these correlations were converted to $Z^{\prime}$ scores following Fisher's $r$ to $Z^{\prime}$ transformation. Such a transformation is necessary when the population value of the correlation coefficient is expected to be other than zero (Hays, 1963). These distributions of $Z^{\prime}$ scores were then compared with a dependent $t$ test. With respect to the first research hypothesis, two nonorthogonal comparisons are relevant. Of particular interest is the comparison between the Normative Regression model and the Functional Subjective model. This statistically significant difference $(t(46)=3.88, p<.01)$ shows that the appropriate scaling of the stimulus dimensions result in a subjective weight model that better reproduces the

\section{Figure 1}

Percentage of Decision Variance Accounted for as a Function of the Two Scaling Procedures and the Two Weighting Strategies Employed in Experiment 1

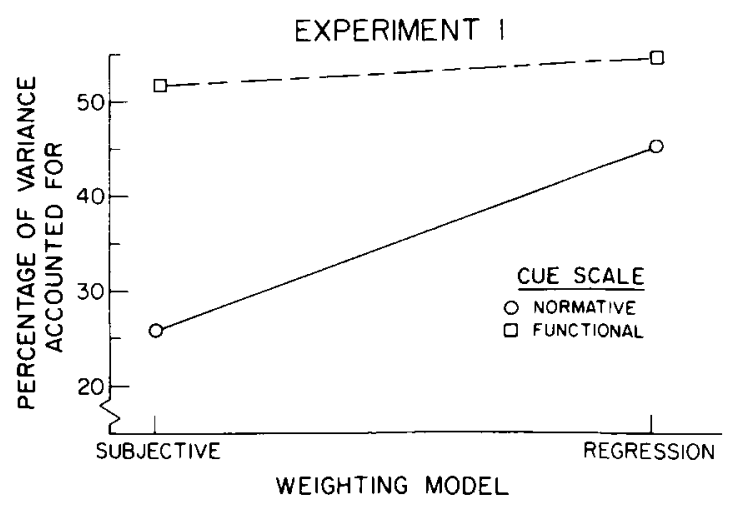


decision maker's judgments than does a regression model employing normative cue scaling. Once the psychological experience in evaluating the stimuli is considered, the utility of the subjective weights becomes apparent and exceeds the Normative Regression model.

The second important comparison involves the two models utilizing functionally scaled predictor values. The difference between the Functional Subjective and the Functional Regression models is not significant (via $t$ test). This finding suggests that although regression models utilizing unscaled predictor cues may be superior to subjective weight models employing unscaled predictor cues, the difference is minimized when each individual's personal scaling of the stimuli is taken into account. Since both models perform similarly in reproducing the decision maker's judgments, there does not seem to be any basis for preferring one over the other in terms of generating inferences about the decision strategy.

Also of interest in the present experiment was whether the use of individually scaled predictor cues would reduce the often reported differences in description of decision strategy as inferred from a consideration of subjective and statistical weights. To facilitate the comparison of subjective weights with regression weights, all betas were transformed to a metric referred to as relative weights, as recommended by Hoffman (1960). For each decision maker the relative weight of each predictor cue was computed according to the formula

$$
R W=100 \mathrm{Br} / \mathrm{R}^{2}
$$

where $R W$ is the relative weight for a predictor cue and is a function of the beta weight of that cue $(B)$, as well as the zero-order correlation between the cue and the criterion ( $r$ ), and the squared multiple correlation coefficient associated with the set of predictor cues and criterion $\left(R^{2}\right)$. The relative weight associated with each cue indicates the percentage of explained variance accounted for by that cue. Since the relative weights sum to 100 , they may be compared directly with the subjective weights obtained from the subject which also sum to 100 , and may be tested statistically via Hotelling's $T^{2}$.
Examination of Figure 2 shows that although the difference between subjective weights and relative weights is reduced when relative weights are defined via functionally scaled stimuli $\left(T^{2}=120.6\right.$, $F=17.9, p<.001)$ rather than normatively scaled stimuli $\left(T^{2}=128.6, F=19.1, p<.001\right)$, the change is minimal. Even for the case in which the predictor dimensions are scaled for each decision maker, major differences in the profiles of subjective and regression weights are obtained.

\section{Experiment 2}

In contrast to the methodology employed by policy-capturing researchers, the results of the first experiment has shown that the use of predictor cues scaled for each decision maker results in a decision model that performs much better in terms of reproducing the decision maker's judgments. In Experiment 1 , the subjective scale values were obtained indirectly from the decision maker using a mathematical procedure.

\section{Figure 2}

A Comparison of the Subjective Weighting Strategy with the Two Statistical Weighting Strategies Obtained Using Scaled and Unscaled Predictor Cue Levels $(\mathrm{SAL}=$ Salary, $\mathrm{SCH}=$ Schedule, DUR $=$ Project Duration, SIZ = Size of Work Group,

$\mathrm{ADV}=$ Opportunity for Advancement, DEV = Potential for Professional Development)

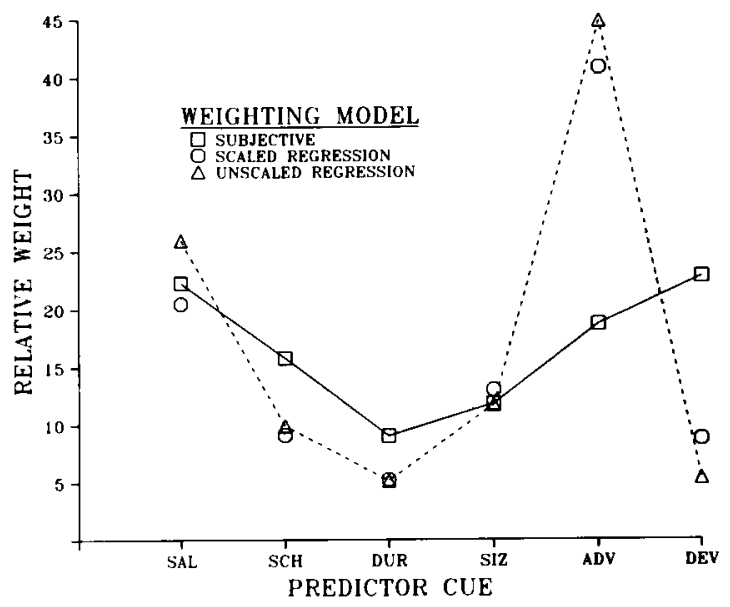


In Experiment 2, two different models were employed to estimate the subjective stimulus scales for each decision maker. One of the scales was computed from a mathematical analysis of the decision maker's judgments, while the second was based on the ratings of the levels of each cue dimension obtained from the decision maker. If both cue scales result in a similar description of judgment strategy, it can be argued that both the rating data and mathematically derived scales provide a reasonably equivalent estimate of the subjective value of the stimulus cues.

\section{Method}

Subjects. Forty-six subjects enrolled in an introductory psychology class at the University of California, Riverside, participated in the test and retest phases of the experiment. The two sessions required about 1 hour each and were separated by 1 week. Subjects were tested in groups and received course credit for their participation in the experiment.

Decision task. Subjects rated hypothetical dating partners on the basis of five predictor cue dimensions. These consisted of intelligence, physical attractiveness, social status, religiosity, and dating activity. Within these five dimensions five levels were selected such that they represented degrees of the attribute that might be encountered in a potential dating partner. Figure 3 provides a sample description and a response scale.

Procedure. The experiment consisted of two sessions, the second being an exact replication of the first. In each session subjects were provided with a booklet of stimulus materials similar to that employed by Zedeck (1977) and were given a brief description of the decision task by the experimenter. A more complete set of instructions was contained in the test booklet.

Prior to the judgment task, subjects rank ordered the five levels of each cue dimension according to their willingness to date a person with that attribute (with a " 1 " indicating a person he or she would not like to date and with a " 5 " indicating a person that he or she would like to date very much). The levels for each cue dimension were presented on a single page, one above the other, and ordered randomly.

After completing the rating task described above, 100 experimental trials were presented. For each of these trials, subjects read a paragraph consisting of one level of each of the five predictor cues and indicated how willing he or she would be to date the person described on a 1 to 5 scale (where a " 1 " indicated someone he or she would not like to date, and a " 5 "' indicated someone they would like to date very much). The distribution of stimulus cues was rectangular, and the intercorrelations among the predictor pairs was exactly zero.

After completing the judgment task, the five predictor cue dimensions were listed on a single page and subjects were instructed to allocate a total of 100 points according to the relative importance, or weight, of that cue dimension in their evaluation of a hypothetical dating partner.

\section{Figure 3}

An Example of One of the 100 Stimulus

Paragraphs Presented in Experiment 2

This person is physically attractive. She comes from an upper middle class background and enjoys going out one weekend a month. Based on various measures, she exhibits a slightly below average intelligence. She is very religious.

\section{DEFINITELY WOULD NOT LIKE TO DATE}

DEFINITELY

WOULD LIKE TO DATE 
Stimulus scaling. Four different procedures were employed to scale the predictor cues. The four predictor cue scales developed were

1. A Priori scale: Each of the five levels of the five predictor cue dimensions generated by the experimenter were assigned values 1 to 5 on the basis of the cue attribute's relative location along the cue dimension (i.e., for the intelligence cue dimension a " 1 " was assigned to the level of "below average," while a " 5 ", was assigned to the level of "genius").

2. Rating scale: The individual ratings, or rank ordering, of the levels of each cue dimension presented prior to the presentation of the stimulus paragraphs were employed as scale values for each of the five predictor cue dimensions.

3. Normative cue scale: A Normative cue scale for the 46 subjects in the experiment was generated by computing the mean ratings associated with each of the cue levels for a given cue dimension. Within each cue dimension these mean values were then rank ordered and assigned the values 1 to 5 , where a value of " 1 ",

\section{Figure 4}

Normative Predictor Cue Scales Plotted as a Function of the A Priori Cue Scale Values for the Five Cue Dimensions Employed in

\section{Experiment 2}

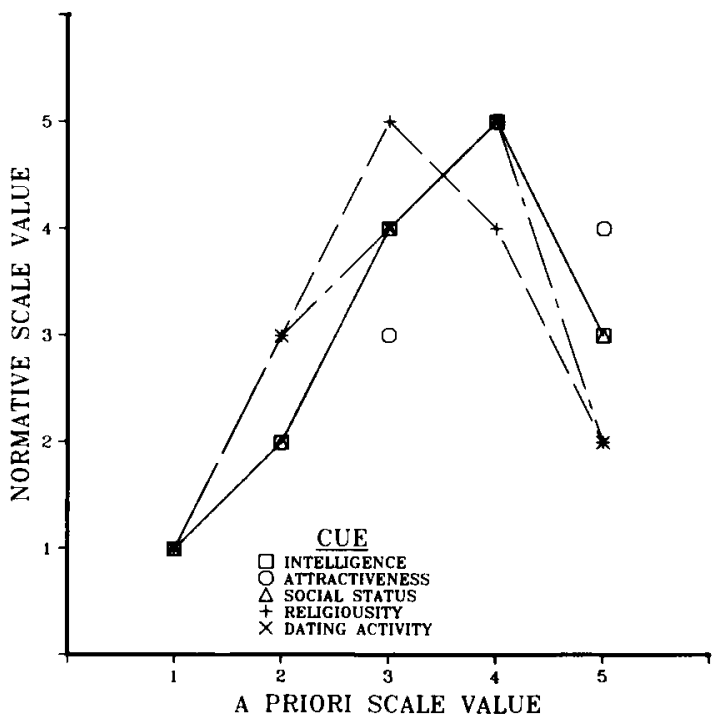

was associated with a relatively unfavorable attribute and a " 5 " corresponded to an attribute that the group found very desirable in a dating partner. Comparisons of the A Priori scale and Normative cue scale values are shown in Figure 4.

4. Functional cue scale: A Functional cue scale for each subject in the experiment was computed by calculating the mean value of the decision maker's judgments across the 100 paragraphs for each level of the five predictor cues. Within each cue dimension these mean values were then rank ordered and were assigned the values 1 to 5 , where a value of " 1 " was associated with a relatively unfavorable attribute and a " 5 ", corresponded to an attribute that the decision maker found very desirable in a dating partner.

Weighting strategy. As in the previous experiment, two different weighting models were compared. In one the points allocated to the five predictor cues were used as estimates of relative importance, or weight, in the linear model. Estimates of relative importance were also calculated by a least squares regression analysis of the predictor cues and resultant judgments.

\section{Results and Discussion}

Eight different decision models were developed for each subject (four types of stimulus scales crossed with two weighting strategies). Each model was employed to predict the ratings obtained for the 100 stimulus paragraphs in the retest phase of the experiment, and the zero-order correlation between these predicted values and the subjects' judgments were calculated. The mean proportion of variance accounted for by each of these models is shown in Figure 5. These correlations were converted to $Z^{\prime}$ scores following Fisher's $r$ to $Z^{\prime}$ transformation, and a repeated measures ANOVA (predictor cue scale by weighting strategy) was performed. The results of this analysis showed a significant main effect for cue scale $(F(3,135)=25.3, p<.001)$ as well as a significant main effect for weighting strategy $(F(1,45)=34.7, p<.001)$ and a sig- 


\section{Figure 5}

Mean Percentage of Decision Variance Accounted for as a Function of the Four Scaling Procedures and Two Weighting Strategies Employed in Experiment 2

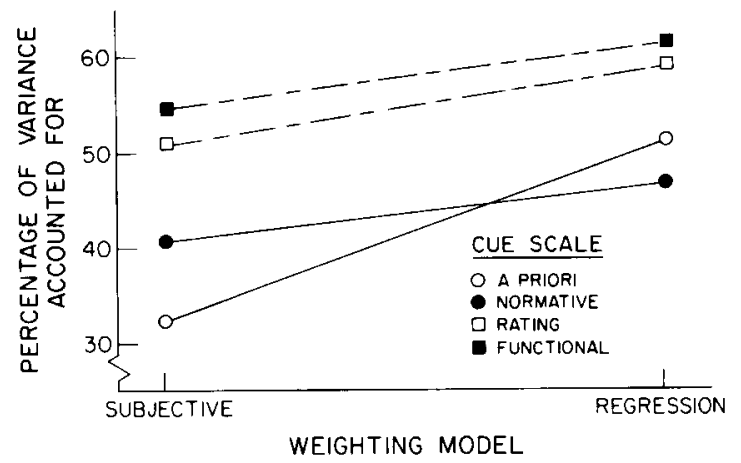

nificant interaction between these two variables $(F(3,135)=19.1 p<.001)$.

An analysis of these same data allows for the consideration of five different decision strategies for each decision maker. Four of these models employ relative weights (Hoffman, 1960) derived from a consideration of the beta weights and multiple correlations associated with each of the regression weight models, while the fifth model of decision strategy was determined directly from the points allocated by each decision maker to the five predictor cue dimensions. The mean weightings associated with each of these descriptions of the decision strategies are shown in Figure 6.

Again, it was of interest to determine whether the use of subjectively scaled predictor cues would eliminate the discrepancy generally reported in the literature between the descriptions of decision strategy obtained directly from the decision maker and those inferred from a regression analysis of the predictor cues and criterion judgments. This hypothesis was tested with Hotelling's $T^{2}$ test comparing the subjective weight model with the Normative model $\left(T^{2}=57.26, F(5,41)=10.43\right.$, $p<.001)$, the Rating model $\left(T^{2}=63.27\right.$, $F(5,41)=11.53, p<.001)$, as well as the Functional model $\left(T^{2}=47.58, F(5,41)=8.67\right.$, $p<.001)$. The hypothesis that the differences between the subjective and regression weight models could be explained wholly in terms of stimulus scaling was not supported by the data of the present experiment.

One other comparison between the descriptions of weighting strategy is of particular interest. It was hypothesized that the Functional and Rating scaling procedures would yield equivalent estimates of the subjective scale values and, accordingly, identical descriptions of the decision strategy. However, a comparison of these two models revealed that they differed significantly $\left(T^{2}=41.95\right.$, $F(5,41)=7.65, p<.001)$ in their descriptions of the decision strategy. Although such a finding seems inconsistent with the experimental hypothesis, a consideration of the procedure used to generate the scales allows for a more guided conclusion. The Functional scale values were assigned on the basis of the mean judgments produced by the decision maker for the five levels of each predictor cue. For predictor cues contributing very little to the ultimate judgment, it is to be expected that the scaling of cue value would tend to be unreliable. For the extreme case in which a cue dimension contributed nothing to the judgment, the procedure by which numbers are assigned to the cue levels must, of course, be irrelevant. Similarly, it may be that decision makers do poorly in ranking cues that they consider unimportant in evaluating a hypothetical dating partner. Such an analysis suggests that a high level of agreement would be expected for relatively important cues, but the weight inferred about cues of lesser importance may differ as a function of the procedure employed to derive the subjective stimulus scales. The differences in relative weights for the models based on the Rating and Functional scaling was tested by performing a dependent $t$ test for each predictor cue dimension. It appears that the overall significant difference between the two descriptions of weighting strategy was produced almost entirely as a result of differences in the specification of the weight of the social status cue $(t(45)=-5.69, p<.001)$. Although the two scaling procedures generated relative weights whose means differed by only 1.76 units, the Functional scale very reliably overestimated the weight of the social status cue compared to the weight assigned by the Rating scale procedure. The dif- 
Figure 6

A Comparison of the Subjective Weighting Strategy with the Four Weighting Strategies Computed from a Regression Analysis of the Predictor Cue Scale Values and Criterion Judgments

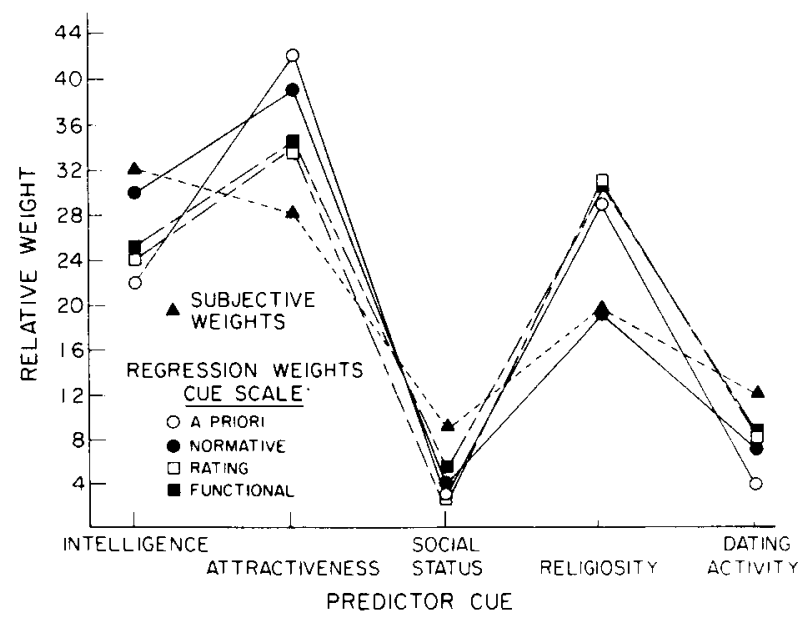

ferences in relative weights determined by the two scaling procedures was found to be marginally significant for one other predictor cue. For this case it was found that the Rating scaling procedure tended to overestimate the weight of the religiosity cue $(t(45)=2.07, p<.05)$. Once again, the mean difference in weight assigned to this cue was extremely small (1.11 units).

In this experiment two different methods were employed to scale the stimulus cues for each decision maker. For the Rating scale the scale value of each predictor cue was assigned on the basis of information obtained directly from the subject, while for the Functional scale the scale values for each cue were inferred from a consideration of the responses produced in the judgment task. Models based on either of these two scales better reproduced the decision makers' judgments than did those models using the Normative scale (see Figure 5). Obviously not all (and possibly none) of the decision makers experienced the stimulus cues exactly as specified by the Normative scale, and when these inappropriate cue scales were employed to model the judgments obtained for the decision task, the resultant decision model performed poorly. This certainly suggests that in modeling decision mak- ing, the researcher must not assign stimulus values on the basis of personal beliefs about norms, or by the location of a particular stimulus along a physical continuum. More importantly, the studies presented in this paper show that even a Normative cue scale is inappropriate.

Aside from the question of how well the different scaling procedures can reproduce the product of the decision processes, what do the models tell about the nature of the processes themselves? Each of the models seem to show that the cue attributes of intelligence and physical attractiveness play an important role in rating the perceived desirability of a hypothetical dating partner, whereas the social status cue and dating activity cues do not. While the religiosity cue appears to be of intermediate importance in evaluating hypothetical dating partners, the weight associated with this cue varies from moderate to high, as a function of the decision model under consideration.

In the absence of any external criterion, it is impossible to specify which, if any, of the descriptions of judgment strategy adequately represent the underlying decision processes. However, an inspection of Figure 6 reveals a remarkable correspondence between the weighting strategies deter- 
mined by the Rating and Functional scaling procedures. Such a similarity in description of judgment strategy is possible only if both procedures yield corresponding estimates of the subjective experience associated with the levels of each predictor cue dimension. Of course, it might be argued that simply because the two procedures produce similar estimates, it does not automatically follow that either are appropriate estimates of the subjective experience associated with the stimulus cues. This argument does not seem tenable, however, when the dissimilar nature of the tasks employed to produce the scales are considered. Since a similar subjective scale resulted from two convergent operations, and the use of this type of scale resulted in a decision model that performed much better in reproducing the decision makers judgments, it seems reasonable to conclude that the use of either procedure yield an appropriate specification of the subjective experience associated with the stimulus cues. It is only through the use of procedures such as these for specifying predictor information that sense can be made out of the specific weights decision makers place on the cues in forming their composite judgments.

\section{Discussion}

The two studies presented in this paper confirm the hypothesis that the scaling of stimulus cues for each decision maker results in a judgment model that is much more successful in reproducing the decision maker's responses than models employing the same cue scales for all decision makers. The pattern of results obtained from these experiments was extremely consistent, even though quite dissimilar procedures were employed to obtain the scales.

One procedure required the subjects to rank order the levels of each cue dimension, and then these ratings were employed as the scale values of the stimulus cues. Another procedure required the stimulus cues to be scaled on the basis of average judgments produced in the actual decision task. Despite the dissimilar nature of the scaling procedures, the use of both procedures resulted in a more predictive judgment model than did models employing the Normative cue scale values for all decision makers. The finding is even more striking, since the Normative cue scales were developed from responses obtained from the same sample of decision makers to which they would be applied. Clearly, if optimizing the product of the decision processes is desired, then for each decision maker the subjective experience associated with the stimulus cues must be considered. The use of Normative cue scales in decision modeling will always be deficient to the extent that any decision maker's experience differs from the normative values.

The results of the present experiments also help explain the disparate reports about the performance of the subjective vs. regression weight models in reproducing the decision maker's judgments. Several researchers have compared the performance of the two models and have concluded that the regression model is the more adequate decision model (e.g., Permut, 1973; Slovic et al., 1972; Slovic \& Lichtenstein, 1971; Summers et al., 1970; Zedeck, 1977). This conclusion is evidently based on some early empirical data indicating a clear superiority for the regression weight model with respect to a prediction criterion. On the other hand, several other investigators have recently reported that the performance of the subjective weight model is more comparable to that of the regression weight model (e.g., Cook \& Stewart, 1975; Jacobs \& Cotton, 1979; Schmitt, 1978). Even though most of these studies have reported that the regression weight model performs better than the subjective weight model, the difference is not large. Although the latter studies employed procedures designed to minimize a statistical artifact favoring the regression model, a comparison of the relative performance of the various weighting models of Experiment 2 suggests an additional explanation for these conflicting reports.

When the scaling of the stimulus cues is clearly not representative of the decision maker's subjective experience (as in the case of the A Priori stimulus scales for many decision makers), the subjective weight model will almost certainly be quite inferior to the regression weight model. This differential performance is obtained because the subjective weights are specified without regard to the 
decision maker's judgments and are thus fixed regardless of whether an appropriate or inappropriate stimulus scale is to be employed. On the other hand, the regression weight model can compensate for even poor stimulus scaling by generating the least squares best fit between the stimulus scale and the obtained judgments. As such, it is hardly surprising that when inappropriate stimulus scales are employed in the modeling procedure, it is found that the regression model performs much better than the subjective weight model, even when both models are cross validated.

A somewhat different finding is obtained when more adequate procedures for stimulus scaling are employed (i.e., the Normative scale to some extent and, certainly, the various stimulus scales developed for each decision maker). For these cases the subjective weight models perform more comparably with the regression weight model. With regard to the results of the present investigation, it is suggested that the magnitude of the differential performance between the subjective and regression weighting models may be more indicative of the adequacy of the researcher's scaling procedures than an indicator of which model best describes the underlying decision processes.

The differences in interpretations that result from the use of normatively vs. individually scaled predictors may have important consequences in the manner such information is used. For instance, Zedeck (1977) has advocated an informationprocessing analysis of motivation in which organizations offer benefits based on employee preferences for organizational rewards. He suggests that the "cafeteria style reward plans . . . in which employees can choose their own benefit packages, could more easily be implemented if organizations understood the real preferences and priorities of their employees"' (p. 75). Presumably, these real preferences would be based on a regression analysis rather than those offered by the employee. In light of previous studies, such an interpretation might be defended. However, the results of the present studies suggest that there is no compelling statistical basis for the adoption of the regression model over the subjective model. As such, the subjective model may in fact be the more attractive of the two, since it allows the individual the greatest opportunity to exercise control over his or her own rewards.

Although it was the original intent of this investigation to better understand the discrepancy between the profiles of weights obtained via different methods of analysis, this study still leaves many questions unanswered. Why are different descriptions for decision processes still obtained dependent on the procedures for obtaining these weights? What are the practical consequences of the differing profiles? Which, if any, of the explanations of process are ultimately related to the decision rendered and subsequent real world behavior? Clearly, further research is needed to help understand the nature of the difference in variable weighting. It is hoped that this investigation has pointed out the need to look not only at the problems associated with variable weighting but also at problems that may precede the combination of information such as the perception of stimulus values.

In summary, it is suggested that stimulus scaling at the level of the individual subject will result in policy-capturing models with more predictive power. Further, this study has shown that the relative performances of models based on regression weights and models which employ weights generated by the subject are heavily dependent on methodological variables. As such, it cannot reasonably be concluded at this time that people are unaware of the manner in which they use information and that, if there is to be an understanding of the way people utilize information, there must not only be concern about variable weighting but also consideration of the subjective experience the individual decision maker has with respect to the levels of each variable.

\section{References}

Anderson, N. H. Functional measurement and psychological judgment. Psychological Review, 1970, 77, 153-170.

Anderson, N. H. Integration theory and attitude change. Psychological Review, 1971, 78, 171-206.

Anderson, N. H. Looking for configurality in clinical judgment. Psychological Bulletin, 1972, 78, 93-102. 
Anderson, N. H. Algebraic models in perception. In E. C. Caterette \& M. P. Friedman (Eds.), Handbook of perception (Vol. 2). New York: Academic Press, 1974.

Anderson, N. H., \& Shanteau, J. Weak inference with linear models. Psychological Bulletin, 1977, 84, 11551170.

Cook, R. L., \& Stewart, T. R. A comparison of seven methods for obtaining subjective descriptions of judgmental policy. Organizational Behavior and Human Performance, 1975, 13, 31-45.

Darlington, R. B. Multiple regression in psychological research and practice. Psychological Bulletin, 1968, $69,161-182$.

Hays, W. L. Statistics for psychologists. New York: Holt, Rinehart, \& Winston, 1963.

Hoffman, P. J. The paramorphic representation of clinical judgment. Psychological Bulletin, 1960, 47, $116-$ 131 .

Jacobs, R., \& Cotton, B. Policy-capturing results: Meaningful differences or differences in meaning. $\mathrm{Pa}-$ per presented at the meeting of the Western Psychological Association, San Diego CA, April 1979.

Kerlinger, F. N., \& Pedhazur, E. J. Multiple regression in behavioral research. New York: Holt, Rinehart, \& Winston, 1973.

Nunnally, J. C. Psychometric theory. New York: McGraw-Hill, 1967.

Nystedt, L., \& Magnusson, D. Integration of information in a clinical judgment task, an empirical comparison of six models. Perceptual and Motor Skills, $1975,40,343-356$.

Permut, S. E. Cue utilization patterns in student-faculty evaluations. Journal of Psychology, 1973, 83, 41-48.

Schmitt, S. Comparison of subjective and objective weighting strategies in changing task situations. Organizational Behavior and Human Performance, 1978, 21, 171-188.

Schmitt, N., \& Levine, R. L. Statistical and subjective weights: Some problems and proposals. Organiza- tional Behavior and Human Performance, 1977, 20, 15-30.

Slovic, P. Analyzing the expert judge: A descriptive study of a stockbroker's decision processes. Journal of Applied Psychology, 1969, 53, 255-263.

Slovic, P., Fischhoff, B., \& Lichtenstein, S. Behavorial decision theory. Annual Review of Psychology, 1977, 28, 1-39.

Slovic, P., Fleissner, D., \& Bauman, W. S. Quantitative analysis of investment decisions. Journal of Business, 1972, 45, 283-301.

Slovic, P., \& Lichtenstein, S. C. Comparison of Bayesian and regression approaches to the study of information processing in judgment. Organizational Behavior and Human Performance, 1971, 6, 649-744.

Summers, D. A., Taliaferro, J. D., \& Fletcher, D. J. Subjective vs. objective description of judgment policy. Psychonomic Science, 1970, 18, 249-250.

Wiggins, N. Individual differences in human judgment: A multivariate approach. In L. Rapport \& D. A. Summers, Human judgment and social interaction, New York: Holt, Rinehart, \& Winston, 1973.

Zedeck, S. An information processing model and approach to the study of motivation. Organizational Behavior and Human Performance, 1977, 18, 47-77.

\section{Author's Address}

Send requests for reprints or further information to Bill Cotton, Bell Laboratories 3D-510, Holmdel NJ 07733, U.S.A.

\section{Acknowledgments}

The authors thank Arlo K. Myers and Ovid J. L. Tzeng for their careful and insightful comments on this manuscript. 